\title{
Entre Mars et Vénus. Le genre de la danse en Italie au $\mathrm{XV}^{\mathrm{e}}$ siècle
}

Between Mars and Venus: genre of dance among the Italian dance-masters of $15^{\text {th }}$ century

\section{Ludmila Acone}

\section{(2) OpenEdition}

Journals

Édition électronique

URL : https://journals.openedition.org/clio/13736

DOI : 10.4000/clio.13736

ISSN : 1777-5299

Éditeur

Belin

Édition imprimée

Date de publication : 1 décembre 2017

Pagination : 135-148

ISBN : 978-2-410-00859-3

ISSN : 1252-7017

Référence électronique

Ludmila Acone, "Entre Mars et Vénus. Le genre de la danse en Italie au xve siècle », Clio. Femmes, Genre, Histoire [En ligne], 46 | 2017, mis en ligne le 01 décembre 2020, consulté le 29 avril 2022. URL : http://journals.openedition.org/clio/13736 ; DOI : https://doi.org/10.4000/clio.13736 


\title{
Entre Mars et Vénus \\ Le genre de la danse en Italie au $\mathrm{XV}^{\mathrm{e}}$ siècle
}

\author{
Ludmila ACONE
}

E poi venghino incontro l'una all'altro con un passo doppio

Guillaume le Juif ${ }^{1}$

Ainsi Laurent de Médicis décrit-il dans sa chorégraphie Venus, la rencontre entre l'homme et la femme. La danse, cet exercice assez noble pour qu'il nous en ait laissé deux chorégraphies, cette scientia liberale comme la décrit le maître à danser Domenico da Piacenza, célèbre la rencontre entre homme et femme qui s'exprime également dans les représentations de Mars et Vénus omniprésentes dans l'expression artistique du Quattrocento. Avec l'organisation et la théorisation de la danse noble dans l'Italie du XVe siècle, les fondements de la danse, son exécution et sa pratique font l'objet d'une analyse méthodique fondée sur une théorie du mouvement et sur la définition d'une bienséance alliant la technique et les codes du cérémonial des cours. Parallèlement à un effort de formalisation technique et pédagogique, les maîtres à danser du Quattrocento définissent des comportements fortement genrés où hommes et femmes assument des rôles précis bien différenciés. Faut-il rappeler que les nombreuses condamnations ecclésiastiques et morales de la danse dénoncent particulièrement sa lascivité renvoyant à une

1 «Et puis qu'elles aillent l'une vers l'autre en faisant un pas double » [trad. de l'A.]. Nous reprenons ici la forme française traditionnelle, traduction du nom italien Guglielmo Ebreo da Pesaro ; à la suite de son baptême (entre 1463 et 1465) celui-ci prend le nom de Giovanni Ambrosio. Zambrini 1873. 
caractérisation "féminine" et conduisant au péché de luxure². Mais au $\mathrm{XV}^{\mathrm{e}}$ siècle, avec l'émergence des cours, il convient de règlementer le comportement des jeunes nobles et de les éduquer afin que l'image de la cour au sein de la cité réponde à un idéal de vertu et de perfection. Comment la danse du Quattrocento théorisée par des maittres à danser met-elle en scène les différences entre le masculin et le féminin? Quel est le lien entre la manière de danser et ce qu'on appellerait aujourd'hui une assignation genrée ? L'élément masculin ou l'élément féminin possèdent-ils des vertus positives qui leur sont propres et/ou respectivement supérieures à celles de l'autre? Comment ce modèle de différenciation s'exprime-t-il à travers le thème de la concordia discors et de l'opposition entre Mars et Vénus ?

Après des années 1980 fondatrices de la recherche sur la danse de la Renaissance ${ }^{3}$, les années 2000 ont vu se développer des recherches portant plus spécifiquement sur la question de la différenciation des comportements masculins et féminins et de la fonction genrée de la danse dans l'Italie des $\mathrm{XV}^{\mathrm{e}}$ et $\mathrm{XVI}^{\mathrm{e}}$ siècles ${ }^{4}$. Cette question est cependant abordée presque uniquement du point de vue de la définition du comportement féminin ${ }^{5}$. La construction de la masculinité en danse pour cette période historique reste un terrain de recherche à défricher.

Quelques aspects de la fonction genrée de cette discipline dans les cours italiennes du Quattrocento à travers les traités de danse sont révélateurs, notamment l'éducation par la danse, la bienséance de cour et la théorisation de l'opposition entre Mars et Vénus

2 Voir Acone 2015a.

3 Un premier point d'orgue est, en 1987, l'organisation d'un colloque international consacré à Guglielmo Ebreo da Pesaro et à la danse de son époque (Padovan 1990). Citons également les travaux pionniers du musicologue Franco Alberto Gallo (Gallo 1979 et 1992) ainsi que Pontremoli \& La Rocca (1987).

4 Cf. David e Salomé (Arcangeli 2001) ; Jennifer Nevile (Nevile 2005); Eugenia Casini Ropa et Francesca Bortoletti (Casini-Ropa \& Bortoletti 2007) ; Alessandro Pontremoli (Pontremoli 2011); Acone 2013.

5 Barbara Sparti développe cette question en particulier dans ses travaux sur Isabelle d'Este (Sparti 2007). En France, Marina Nordera organise au Centre national de la danse en 2004 une exposition qui a fait date sur « La construction de la féminité dans la danse » (Nordera 2004). 
- opposition entre vertu guerrière masculine et beauté et douceur féminine propres à la Renaissance italienne.

\section{La danse, un fondement de l'éducation des nobles}

$\mathrm{Si}$, comme l'affirme Balthazar Castiglione, "la principale et vraie profession du Courtisan doit être celle des armes $»^{6}$, celui-ci, doit également faire preuve de maitrise et de perfection dans tout exercice corporel :

De même, dans la danse, un seul pas, un seul mouvement du corps, fait avec grâce et sans être forcé manifeste aussitôt le savoir de celui qui danse $\mathrm{T}^{7}$.

L'homme de cour fait appel pour cela à un maitre à danser qui se met au service des cours et qui, pour ses représentants les plus illustres, devient également un théoricien de son $\operatorname{art}^{8}$. Le maittre à danser devient un personnage important qui contribue à la bonne éducation des jeunes hommes et jeunes filles nobles. Il faisait partie des familiers d'une princesse et la suivait dans ses déplacements. À Venise, quand la fiancée exécutait le pas de danse que lui imposait la coutume, elle était conduite, à défaut de son père, par son maître à danser que l'on choisissait généralement d'âge mûr: s'il pouvait remplacer le père dans une occasion telle que le mariage, la bienséance voulait cependant qu'il ne fût pas trop jeune afin de protéger la réputation de la jeune épouse?

La danse constituait également un élément important dans la politique matrimoniale des familles. Tous les traités relatifs à l'éducation des jeunes femmes placent au premier rang l'art de la

6 Castiglione $1987: 42$.

7 Castiglione $1987: 58$.

8 Les plus célèbres sont Domenico da Piacenza (ca 1390 - ca 1470), qui écrit vers 1455 le premier traité de danse connu en Occident (Bianchi 1963) et ses deux disciples, Guillaume le Juif (1420 - ca 1484) (plusieurs exemplaires connus et édités dont Zambrini 1873 et Messori Roncaglia 1885) et Antonio Cornazzano (1429 - avant 1500) (Mazzi 1915). Pour l'ensemble des enjeux concernant les traités et la théorisation de la danse au XVe siècle voir Acone 2013.

9 Sanudo $1997: 445$. 
danse et celui du chant qui permettaient d'évaluer leur bonne éducation. Lorsque Alessandra de' Macinghi Strozzi, d'une famille de riches marchands florentins, dut faire face à l'exil de ses fils par la ville de Florence, elle déploya des efforts considérables pour qu'ils retrouvent leur statut et chercha pour l'aîné une épouse digne de son rang. En 1465, elle lui écrivait pour lui vanter les attraits d'une jeune personne qu'elle souhaitait lui voir épouser :

J'ai demandé si elle était gauche et empruntée, on m'a répondu que non, qu'elle était au contraire fort adroite, car elle savait danser et chanter. S’il en est ainsi sur ce point, je ne doute pas du reste ${ }^{10}$.

La bonne danseuse apparaît ainsi comme le bon parti.

\section{Esthétique de la danse noble et comportement féminin}

Si durant le Quattrocento, hommes et femmes se côtoient en dansant, c'est à condition de respecter diverses normes : la virtuosité et l'agilité sont préconisées chez l'homme, les femmes doivent se montrer plus mesurées. Antonio Cornazzano précise qu'elles ne conviennent pas aux dames, sauf à la danser posément et sans s'essayer à des sauts et des prouesses incongrues :

Mais s'il lui arrive de danser [la pive], ne conviennent à la femme que ses pas naturels et son aide à l'homme dans ses tours, selon les ronds de jambe ou les sauts qu'il fera, droits ou bien renversés en dedans ou en dehors ; et il faut qu'elle soit rapide et bien adroite car la mesure [de cette danse] vole plus vite que les autres. [...] la femme ne doit à aucun moment se détacher du sol ${ }^{11}$.

Dans leurs traités, Domenico da Piacenza, Antonio Cornazzano et Guillaume le Juif ne se contentent pas d'émettre des préceptes moraux, ils visent plus généralement à établir une grammaire, une langue de leur discipline. La théorisation de la danse, la fixation de principes précis et ordonnés deviennent nécessaires du fait de la transformation de cet art en un « art libéral » et de son enseignement ; elles accompagnent la professionnalisation des maitres à danser des cours princières. La classification, l'ordre, la fixation de principes fondamentaux sont indispensables à la transmission de cet art, à ses

\footnotetext{
10 Guasti $1877: 445$.

11 Mazzi 1915 : 11.
} 
exigences techniques sophistiquées, mais également à la structuration d'une discipline née dans et pour les cours : au-delà d'un nouveau langage, il s'agit de construire une norme de comportement et de bienséance. Cornazzano le souligne: l'exercice de cet art nobile et pelegrino (de cet art noble et recherché) impose de fuir «l'agitation vulgaire » et de s'éloigner des origines populaires du ballo da villa (de la danse rustique).

\section{Guillaume le Juif, le Capitulum Regulare Mulieris}

Alors que Domenico da Piacenza n'évoque pas spécifiquement la place des femmes, Guillaume le Juif consacre un chapitre, le Regulare mulieris $^{12}$, aux règles qu'elles doivent suivre et notamment la plus grande modestie dont elles doivent faire preuve en dansant: chacun doit occuper une place précise en fonction de son rang et de son sexe :

La femme jeune et vertueuse qui veut prendre plaisir à apprendre cet exercice et cet art doit observer ses règles avec beaucoup plus de modération et d'honnêteté que l'homme. Que sa manière soit douce, modérée et suave, le mouvement de son corps humble et plein de douceur, son maintien digne et noble. Que son appui sur le pied soit léger ${ }^{13}$.

L'élévation propre à l'esthétique de la danse noble lui confère une grande dignité. Guillaume le Juif continue :

Que ses gestes soient bien formés, que son regard ne soit point hautain ni ne vagabonde çà et là comme beaucoup le font; mais que le plus souvent elle regarde honnêtement vers le sol, sans baisser la tête comme beaucoup le font, mais en la redressant devant la personne qui lui fait face comme la nature l'enseigne presque d'elle-même [...] Et qu'à la fin de la danse, alors que l'homme la quitte, elle lui adresse un doux regard et lui fasse une honnête et respectueuse révérence correspondant à celle de l'homme; puis qu'elle aille se reposer en gardant une attitude modeste, observant les défauts éventuels, les actes justes et les mouvements parfaits des autres ${ }^{14}$.

\footnotetext{
12 Messori-Roncaglia 1885 : 21 .

13 Ibid.

14 Ibid.
} 
Cela permet ainsi aux femmes de compenser la faiblesse de leur esprit, inapte, selon Guglielmo, à saisir une vraie « science » et « art libéral »,

D'autant plus que rares sont les femmes qui comprennent parfaitement cette vertu et cet art, car elles pratiquent cet exercice en s'en remettant au hasard, plutôt qu'à une quelconque science qu'elles possèderaient ${ }^{15}$.

Toutes ces recommandations de l'auteur concernent uniquement les femmes et ne trouvent leur équivalent dans aucun autre chapitre du livre.

\section{Cornazzano, de la danse à la guerre}

En Grèce antique, la danse était aussi une pratique d'entraînement et un rituel de conditionnement du guerrier voué au culte des dieux. La pyrrbique, et toutes les danses guerrières que nous connaissons depuis, montrent la « double nature » de la musique et de la danse déjà décrite par Platon. Dans La République, le philosophe fustige les nouveautés des harmonies lydiennes molles et séduisantes et les oppose au noble modèle des anciens, simple et viril. Platon fait état d'au moins deux sortes de danses : l'une «qui représente l'état d'une âme sage dans la prospérité et dans les plaisirs modérés », qu'il qualifie de pacifique ; "Quant à l'autre la guerrière, qui est toute différente de la pacifique, on peut justement l'appeler pyrrhique, puisqu'elle imite les parades par lesquelles on se gare des coups et des traits de toute sorte $»^{16}$.

Au cours des $\mathrm{XV}^{\mathrm{e}}$ et $\mathrm{XVI}^{\mathrm{e}}$ siècles, la danse armée était plutôt vouée au culte des valeurs de la cour et à celui du Prince victorieux. Giambattista Giraldi Cinzio (1504-1573), dans L'Homme de cour ${ }^{17}$, exhorte les jeunes combattants à pratiquer la moresque afin de canaliser leur violence et de s'interdire les combats privés. Il rappelle comment :

Chez les Romains, des prêtres appelés Saliens, en sacrifiant à Mars, leur dieu de la guerre, maniaient les épées et les boucliers en une mesure de temps bonne et appropriée comme nous le voyons faire aujourd'hui dans des moresques, par des mouvements du corps vigoureux et légers en temps de musique ${ }^{18}$.

\footnotetext{
15 Messori-Roncaglia $1885: 22$.

16 Platon 1997 : 279 (livre VII, chapitre XVIII).

17 Giraldi Cinzio 1989 : 35.

18 Giraldi Cinzio 1989 : 35-36.
} 
Les traités spécifiques qui sont consacrés tant au combat qu'à la danse prescrivent la place que ces deux disciplines doivent occuper dans la vie de la cour. Il y apparait clairement que ces deux activités se rejoignent parce qu'elles concernent le corps et son entraînement, et parce que le combat, outre son caractère guerrier, est également présent sous des formes ritualisées qui vont de la joute à la danse armée. Cette dernière participe en même temps à la construction des formes d'une identité masculine courtisane. Celle-ci se définit bel et bien dans une continuité avec les valeurs de la virilité antique, mais en y intégrant également des éléments plus policés.

$\mathrm{Si}$ Antonio Cornazzano ne consacre pas dans son traité de développement spécifique à l'attitude des danseurs hommes et femmes, cet auteur d'une œuvre littéraire vaste et variée a illustré plus particulièrement dans deux autres ouvrages sa vision de l'un et l'autre genres: d'abord dans son De excellentium virorum principibus dédié au duc Francesco Sforza, qui exalte les vertus masculines et en particulier celle du condottiere milanais auprès duquel il a exercé ses talents ; ensuite dans le De mulieribus admirandis ${ }^{19}$. Ces deux ouvrages participent d'une tradition vivace, celle des vies des hommes et des femmes illustres inaugurée par Boccace ${ }^{20}$. Contrairement au poète florentin qui n'a pas de mots assez sévères pour condamner l'inconduite de Vénus, Cornazzano conçoit son ouvrage comme un recueil de vies de femmes singulières sans porter sur elles de jugement moral. Il insiste sur les qualités de Vénus sans pour autant passer sous silence les amours illicites de la déesse. Dans une autre

19 Il se trouve dans le Codex 177 de la Bibliothèque Estense de Modène. Composé de 45 folios et écrit à la fin du siècle, il est cité dans Conor (1960). Après deux chapitres introductifs, Cornazzano y rapporte les vies de onze femmes de l'Antiquité ou de la mythologie avant de faire l'éloge du seul personnage contemporain, Bianca Maria, dédicataire de l'ouvrage, dont il exalte la beauté et les vertus morales.

20 Dans son De Claris Mulieribus et dans le De casibus virorum illustrium qui illustre la vie des hommes remarquables depuis Adam et les vertus qui les ont rendus célèbres, Boccace rappelle l'opposition entre Mars et Vénus. Citons également Vespasiano da Bisticci (1421-1498) qui, à la cour de Federico da Montefeltro à Urbino, écrit une compilation des vies d'hommes illustres (Le vite) puis un Libro delle lodi delle donne (Livre des éloges des femmes). 
œuvre, le De Re militari, un traité d'art militaire, Cornazzano précise sa conception des rapports de genre ${ }^{21}$. Cherchant les origines antiques de l'art militaire, pour lui, seules la Macédoine et Rome ont engendré des hommes aptes à bien exercer cet $\operatorname{art}^{22}$. Quant aux vertus du parfait homme d'armes, Cornazzano regrette la valeur virile des hommes d'antan et fait l'éloge de la vie âpre du combattant qui fuit l'oisiveté et les mollesses.

Aussi, dans la deuxième édition de son traité, Cornazzano semblet-il à ce sujet faire acte de «repentance » concernant son activité de maittre à danser :

Bouillait le sang de ma prime jeunesse

À mon âge j'aurais honte

De ce qu'alors je tenais pour vertu.

$[\ldots]$

Jeune, j'écrivis les fondements de ce métier

Lorsqu'elle lui apprenait cet $\operatorname{art}^{23}$.

Dans le De Militari, rapportant la tradition du séjour d'Alexandre à Babylone, il décrit Vénus qui pervertit Mars dans les plaisirs, l'endort et affaiblit ses défenses :

La légende veut qu'il [Alexandre] adore dans Bagdad Vénus et Bacchus, rejette Mars et Pallas la journée et se repose des nuits où il s'épuise, que le dompteur du monde danse au son des cymbales et que du palais de l'hôte vaincu il fasse une étable pour les putains et les mauvais garçons ${ }^{24}$.

21 Cornazzano 1520. Subdivisé en neuf chapitres, le De Re Militari commence par définir l'art militaire comme l'art le plus honorable auquel le génie humain puisse s'exercer, fleur de la science, honneur suprême d'un prince et de son domaine Les chapitres suivants expliquent de façon très détaillée l'art du combat des soldats comme des chevaliers, les techniques de siège mais aussi l'usage des armes à feu, véritable révolution dans l'art du combat, quoique peu appropriée à l'expression des vertus chevaleresques.

22 Cornazzano $1520: 7$.

23 Mazzi 1915 : «Giovine scrissi quel mistier compito/quando imparando lei l'arte cotale/ad ogni posta mia l'hebbi per dito» (p. 7).

24 Cornazzano 1520: livre 1, chapitre 5: «La fama va che Venere in Baldaco/Adora, e Bromio, Marte abietto, e Palla / posasi il giorno, che in notte se stracco. / domitor del mondo al cimbal balla/ E nel primo palazzo del vinto hoste/ Di puttane, e di putti fa una stalla ». 


\section{Harmonie et concordia discors}

Cornazzano reprend un topos opposant la danse, liée à Vénus, et la guerre, liée à Mars. Dans la tradition humaniste, la déesse de l'amour et le dieu de la guerre sont deux principes opposés. L'union de ces deux pôles, concordia discors ou discordia concors, est une condition nécessaire à la naissance d'Harmonie, fille de Mars et Vénus. La concordia discors a été décrite par Héraclite Pythagore en un mouvement incessant. Vénus, déesse de la génération, est invoquée par Lucrèce dans son célèbre poème, le De Natura rerum, afin qu'elle désarme Mars et que l'esprit de la guerre soit dompté par l'amour et la paix.

Depuis l'Antiquité ce thème court dans la littérature et les arts, attribuant des vertus opposées et complémentaires au masculin et au féminin, tantôt en privilégiant de manière rhétorique l'élément féminin capable de transformer les armes en jouets comme dans le célèbre tableau de Botticelli Vénus et Mars, et tantôt en déplorant l'amollissement des mœurs, synonyme de faiblesse et d'avilissement de la force virile. Dans tous les cas, l'expression de cette concordia discors et les vertus qui y sont célébrées répondent au contexte social et culturel du moment, qu'il s'agisse de promouvoir un idéal de concorde et de paix, ou que la politique de la cour promeuve les vertus de la force virile et du combat. Il ne faut pas oublier que malgré la célébration de la concorde et des vertus des bons gouvernements, les princes des cours italiennes sont des chefs militaires qui tentent d'élargir leur territoire ou de le préserver ${ }^{25}$.

\section{Triomphe de Vénus}

Dans Le Parnasse de Mantegna peint en 1497 pour Isabelle d'Este 26 sept muses, - expressions du triomphe des arts à la cour de Mantoue

25 Si le pouvoir des Médicis (dans une cité oligarchique qui est encore, formellement, une République) repose sur la richesse, les Sforza, les Este, les Montefeltro ont acquis et maintenu leur territoire par la force des armes.

26 Le Parnasse (Musée du Louvre), fait partie d'une série de cinq tableaux, que la marquise de Mantoue, Isabelle d'Este (1474-1539), avait commandé pour décorer son premier studiolo dans le palais ducal de Mantoue. Il contient en germe des thèmes qui sont aussi développés dans les autres panneaux: le triomphe de l'amour spirituel sur l'amour terrestre et la célébration des arts à la cour de Mantoue. Tirés des Métamorphoses d'Ovide, les amours de Mars et Vénus 
grâce à la paix et à l'harmonie du couple ducal et du duché - dansent au son de la lyre d'Apollon sous les yeux de Mars et de Vénus. Un peu plus ancienne, la fresque du Mois d'avril que peint Francesco del Cossa dans le Salone dei mesi du Palais Schifanoia de Ferrare décline le thème des amours de Mars et Vénus ${ }^{27}$. Le Mois d'avril illustre la victoire de l'amour sur les instincts guerriers. Sur un char triomphal tiré par deux cygnes blancs, conforme à l'iconographie des Triomphes de Pétrarque, Vénus domine un Mars en armes, enchaîné et agenouillé devant elle. L'image illustre un instant de paix et de concorde qui fait suite aux victoires militaires du duc et à la consolidation de son pouvoir. Les figures de Mars et Vénus sont convoquées pour célébrer la paix retrouvée et l'affirmation d'une puissance politique, celle de Mars rappelle en outre les vertus guerrières du prince et sa fonction protectrice. Les vertus viriles du condottiere qui a su s'élever au pouvoir doivent se faire oublier dans les moments de paix et de concorde. L'harmonie se fonde sur la capacité du prince à assurer un bon gouvernement sans jamais perdre de vue qu'on le doit à ses vertus. Remarquons que dans le Capitulum generale de son traité, Guillaume le Juif rapproche explicitement les quatre mesures de ses danses (basse danse, saltarelle, pive et quaternaria) des quatre tons de la musique et des quatre éléments ${ }^{28}$.

C'est de la correspondance de ces quatre éléments, mesures et tons que nait l'harmonie, et pas seulement dans la danse, car l'harmonie est au fondement de la vie humaine. C'est cette concordance, ou discordance, qui émeut exécutants et spectateurs d'une danse. Selon Marsile Ficin, Vénus vainc Mars qui ne résiste pas à l'amour ${ }^{29}$.

pouvaient être perçus comme une allusion au couple formé par François II et Isabelle d'Este, mécène et protectrice des muses.

27 Célébrations de la puissance politique du duc et de ses vertus, les fresques du Salon des mois ont été exécutées entre 1468 et 1470 pour Borso d'Este à l'occasion de son investiture comme duc de Ferrare par le Pape Paul II.

28 Messori-Roncaglia 1885 : 19.

29 Gentile 2001 : Lettre à Domenico Beniveni sur les principes de la musique : «In quarto luogo la proporzione sequiterza da cui deriva l'armonia del quarto suono, quasi mediana fra la consonanza e la dissonanza e che fonde alcunché di Marte e di Venere ». Guillaume le Juif inspire et s’inspire de Marsile Ficin, cf. Acone 2015a. 
Cet équilibre, harmonie et concordance des principes, s'inscrit dans un contexte intellectuel dominé par le néoplatonisme mais également dans l'ordre de la bienséance propre aux cours ${ }^{30}$. Il fait écho à l'harmonie sociale et politique où chacun occuperait une place précise, qu'il s'agisse du « lieu naturel » cher à Aristote ou de la place qui lui serait attribuée conformément au platonisme et à sa vision de l'harmonie céleste. Ce n'est pas par hasard si dans toutes les descriptions des fêtes de l'époque auxquelles des maitres à danser ont participé ou contribué directement ou indirectement, une des questions fondamentales concernait l'ordre d'apparition des divers personnages en fonction de leur rang social. De même, un ordre spécifique, dans la danse, attribue en fonction de son sexe un rôle précis à chacun et contribue au maintien de la hiérarchie genrée, sociale, politique et spirituelle des cours et des villes. Tout est ordre et mesure, qu'il s'agisse de la mesure musicale ou dansée, de l'équilibre des proportions du corps, du rang et de la place de chacun au sein d'une organisation sociale et politique hiérarchisée.

Le féminin demeure synonyme de concorde de paix et d'harmonie, le masculin incarne les valeurs guerrières et la force, la place des hommes et des femmes sont donc bien précises et représentent les deux pôles du pouvoir et de la politique des cours : cette concorde est incarnée par Mars et Vénus et se réfère tant à la tradition issue de la Grèce Antique qu'aux Triomphes de Pétrarque chers à Laurent de Médicis ${ }^{31}$ soucieux du maintien d'un pouvoir politique sans cesse contesté et menacé à l'intérieur comme à l'extérieur.

\footnotetext{
30 Cf. Acone 2016.

31 Notons qu'à Florence, aux Offices est toujours présent le meuble figurant les Triomphes de Pétrarque peint par Matteo de Pasti et offert à Pierre de Médicis. Sur ce meuble sont représentés la beauté de Vénus et Mars en armure. Müntz 1894 : 209.
} 


\section{Sources}

Bianchi Dante, 1963, "Un trattati inedito di Domenico da Piacenza », La Bibliofilia, LXV, p. 109-149. 72, BNF, P

Castiglione Balthazar, 1987, Le Livre du Courtisan, traduction française de Gabriel Chappuis, revue par Alain Pons, Paris, Éditions Gérard Lebovici.

Cornazzano Antonio, 1520, De Re Militari, Firenze, Bernardo di Filippo di Giunta fiorentino.

GentiLe Sebastiano, 2001, Le Divine lettere del gran Marsilio Ficino, Roma, Edizioni di Storia e letteratura.

Giraldi Cinzio Giambattista, 1989, L'Uomo di corte: discorso intorno a quello che si conviene a giovane nobile e ben creato nel servire un gran principe, éd. par Walter Moretti, Modena, Mucchi.

Mazzi Claudio, 1915, " "Il Libro dell'arte del danzare” di Antonio Cornazano », La Bibliofilia, rivista dell'arte antica, XVII, p. 1-30 (édition du manuscrit d'Antonio CornazZAno, Biblioteca Apostolica Vaticana, codice Capponi 203, 1455).

Messori-Roncaglia Giovanni, 1885, «Della virtute et arte del danzare e di alcune opportune et necessarie particelle a quela pertinenti. Trascizione di un manoscritto indedito del XV secolo esistente nella Biblioteca Palatina di Modena corredata di note ed appunti », Modena, Imm. Concezione (édition du manuscrit de Guillaume Le Juif, De pratica seu arte tripudii vulgare opusculum, Modena, Biblioteca Estense, Codex Italiano $82 \alpha$ J 94).

Platon, 1997, Les Lois, trad. du grec ancien par Anissa Castel-Bouchouchi. Introduction et notes d'Anissa Castel-Bouchouchi, Paris, Gallimard, coll. « Folio essais ».

SAnudo Marino, 1997, I Diarii, XXXVII, éd. par Paolo MArgaroli, Vicenza, Neri Pozza Editore.

ZAMBrini Francesco, 1873, «Trattato dell'arte del ballo di Guglielmo Ebreo pesarese, testo inedito del secolo XV ", Scelta di curiosità letterarie inedite o rare dal secolo XIII al XVII : dispensa CXXXI (131), Bologna, Gaetano Romagnoli (rééd. Bologne, Forni, 1968).

\section{Bibliographie}

ACONE Ludmila, 2013, "Théorie et pratique de la danse noble dans l'Italie centroseptentrionale au XVe siècle », thèse de doctorat sous la dir. de Jean-Philippe Genet, université de Paris I Panthéon-Sorbonne et en co-tutelle avec l'Università Cattolica del Sacro Cuore de Milan, sous la direction de Mme Paola Ventrone. 
—, 2015a : «Guérir le corps et l'âme par la musique et la danse chez Marsile Ficin et Guillaume le Juif », Chroniques italiennes, 30/2 [http://chroniquesitaliennes.univparis3.fr/PDF/Web30/1.L.Acone.pdf]

—, 2015b, « La danse de David et la figure de Saul dans le théâtre florentin (XVe-XVI siècle », Circé, Histoires, cultures et sociétés, 6 [http://www.revue-circe.uvsq.fr/ladanse-de-david-et-la-figure-de-saul-dans-le-theatre-florentin-xve-xvie-siecle/]

_, 2016, «L'ordine et le mouvement, la danse et les fêtes de mariage. Alliances, cérémonies et représentations allégoriques », dans Aude MAIREY, Solal ABÉLÈS \& Fanny MADELINE (dir.), "Contre-champs ». Études offertes à Jean-Philippe Genet, Paris, Classique Garnier, p. 297-320.

ArCANGELI Alessandro, 2001, David o Salome? Il dibattito europeo sulla danza nella prima età moderna, Roma, Viella, Fondazione Benetton Studi e Ricerche.

Casanova Emilio, 1901, La Donna senese del quattrocento nella vita privata, Siena, tipographia L. Lazzari.

CASini-Ropa Eugenia \& Francesca BortoletTi (dir.), 2007, Danza, cultura e sociétà nel Rinascimento italiano, Macerata, Edizioni Ephemeria.

Conor Fahy, 1960, The "De Mulieribus admirandis » of Antonio Cornazzano, Florence, Olschki.

GALlo Franco Alberto, 1979, "Il "Ballare Lombardo” (circa 1435-1475), I Balli e le Basse danze di Domenico da Piacenza e di Guglielmo da Pesaro », Studi Musicali, VIII/1, p. 61-84.

—, 1992, Musica nel Castello. Trovatori, libri, oratori nelle corti italiane dal XIII al XV secolo, Bologna, Il Mulino.

LuZIO Alessandro \& Rodolpho Renier, 1893, Mantova e Urbino, Torino, L. Roux.

Nevile Jennifer, 2005, The Eloquent Body: dance and bumanist culture in fifteenth-century Italy, Bloomington, Indiana University Press.

MüNTZ Eugène, 1894, «Les plateaux d'accouchées et la peinture sur meubles du XIVe au XVIe siècles ", dans Monuments et mémoires de la Fondation Eugène Piot, Paris, Académie et inscriptions et Belles Lettres.

Nordera Marina, 2004, La Construction de la féminité dans la danse, XVe-XVIIIe siècle, Catalogue de l'exposition, Pantin, Centre national de la danse.

Padovan Maurizio (dir.), 1990, Guglielmo Ebreo da Pesaro e la danza nelle corti italiane del $X V^{\circ}$ sec., actes du colloque international (Pesaro 16-18 juillet 1987), Pise, Pacini.

Pontremoli Alessandro, 2011, Danza e Rinascimento. Culture coreica e "buone maniere" nella società di corte del XV secolo, Macerata, Ephemeria Editrice, coll. i libri dell'Icosaedro. 
Pontremoli Alessandro \& Patrizia LA RoccA, 1987, Il Ballare lombardo. Teoria e prassi corentica nella festa di corte del XV secolo, Milano, Vita e pensiero, coll. « La Citta e lo Spettacolo ».

SPARTI Barbara, 1996, "The function and status of dance in the $15^{\text {th }}$ C. Italian courts », Dance Research, 141, p. 42-61.

—, 2007, "Isabella and the Dancing Este Brides, 1473-1514 », in Lynn M. Brooks (dir.), Women's Work: making dance in Europe before 1800, Madison, University of Wisconsin Press, p. 19-48. 